\title{
Developmental trends in interpolation and its spatial constraints: A comparison of subjective and occluded contours
}

\author{
Bat-Sheva Hadad • Daphne Maurer • Terri L. Lewis
}

Published online: 4 March 2015

(C) The Psychonomic Society, Inc. 2015

\begin{abstract}
We examined interpolation in 6- and 9-year-old children and in adults, in the two most common forms of fragmentation: subjective and partially occluded contours. Experiment 1 examined the effects on adults' interpolation of contour geometry, specifically, the effect of a scale-dependent factor (i.e., retinal size) and a scale-independent factor (i.e., support ratio). For both subjective and partially occluded contours, interpolation was affected more by support ratio than absolute size. However, subjective contours were less precisely interpolated and their interpolation was affected more by support ratio than was the case for partial occlusion. Experiment 2 used a subset of retinal size and support ratio levels in children and adults. Interpolation of both subjective and occluded contours improved significantly with age, with the two types of contours equally affected by spatial constraints during early childhood. However, while interpolation of occluded contours became more precise with age and less dependent on support ratio by adulthood, interpolation of subjective contours was less improved and became even more tied to support ratio in adulthood. The implications of these differential age-related changes in the spatial constraints on interpolation of the two types of contours for the mechanisms of perceptual completion are discussed.
\end{abstract}

Keywords Perceptual completion - Subjective contours · Partially occluded contours · Contour interpolation - Support ratio $\cdot$ Development

\section{B.-S. Hadad $(\bowtie)$}

Edmond J. Safra Brain Research Center, Department of Special Education, University of Haifa, Mount Carmel 31905, Haifa, Israel e-mail: bhadad@univ.haifa.ac.il

D. Maurer $\cdot$ T. L. Lewis

Department of Psychology, Neuroscience \& Behaviour McMaster University, McMaster University, Hamilton, ON L8S 4K1, Canada
Continuous objects often project disparate fragments on the retina as a result of occlusion, shadows, or low-reflectance contrast. Yet, in most cases, humans perceive continuous surfaces and coherent objects. A large body of research has been devoted to uncover the visual mechanisms of contour interpolation mediating this perception of continuity. However, fundamental questions, such as the spatial factors limiting interpolation, whether the same process underlies interpolation in different cases of fragmentation, and the developmental stage at which these interpolation processes become adult-like, remain open. To study these questions, we examined interpolation in 6- and 9-year-old children and in adults, in the two most common forms of fragmentation: subjective contours and partially occluded contours.

In the case of partial occlusion, often referred to as amodal completion, interpolation between distant image fragments behind the occluding surface leads to a complete representation of the occluded object (bottom panel in Fig. 1). A complete representation is often achieved without generating any visually experienced structure in the interpolated area (Michotte, Thines, \& Crabbe, 1964). In the case of subjective contours, also known as modal completion, completion yields a visual impression of contours or surfaces in locations where there is no local image contrast to support this percept (top panel in Fig. 1).

A major question in the adult literature is whether a single, common interpolation mechanism is employed by the visual system to transcend these two forms of fragmentation. Kellman and Shipley argue that the same interpolation process operates without regard to the final appearance of a subjective or an occluded figure (e.g., Kellman \& Shipley, 1991; Yin, Kellman, \& Shipley, 1997). By this identity hypothesis, the appearance of the interpolated contour, as subjective or as occluded, relates to the mechanism responsible for assigning relative depth of the completed contours and surfaces. Empirical support for this hypothesis comes from studies showing, for example, that the two types of contours are affected similarly by contour alignment (Shipley \& Kellman, 1992), that they demonstrate similar sensitivity to shape 


\section{Subjective Contours}

\section{Occluded Contours}

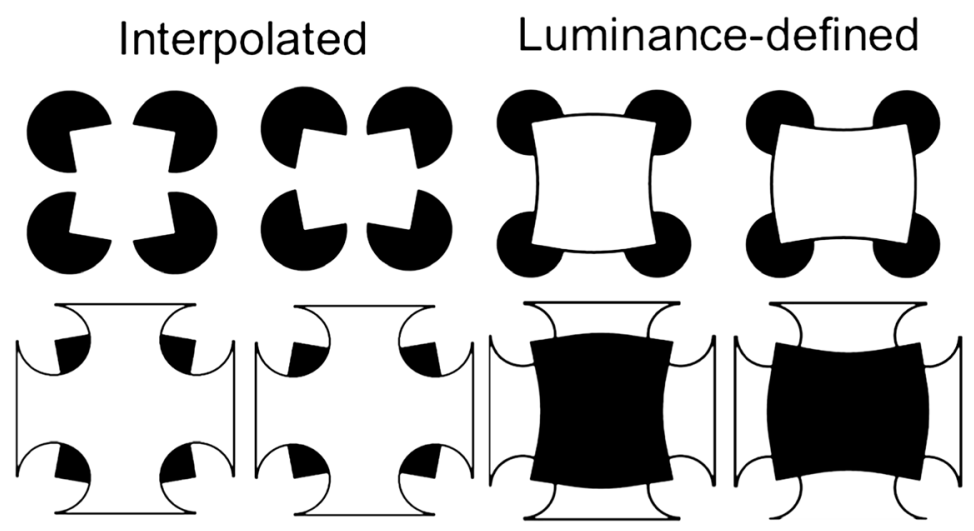

Fig. 1 Subjective (top) and occluded (bottom) interpolated contours. The luminance-defined counterparts of each are presented on the right

changes (Kellman, Yin, \& Shipley, 1998; Ringach \& Shapley, 1996), and that they are obligatory in that they arise even when they are detrimental to performance (Davis \& Driver, 1998; He \& Nakayama, 1992; Rensink \& Enns, 1998). A common neural mechanism, manifesting in a modulation in response strength within the lateral occipital complex (LOC) and higher parietal structures, has also been observed (Murray, Foxe, Javitt, \& Foxe, 2004).

There is, however, other evidence suggesting that two separate mechanisms are involved in the interpolation of subjective and occluded contours. For example, while interpolation of subjective contours depends strongly on the prevailing luminance relationships within a scene, interpolation of occluded contours does not (e.g., Anderson, Singh, \& Fleming, 2002; Nakayama \& Shimojo, 1992). The shape of the interpolated contours may even change when a figure undergoes a transition from a modal to an amodal appearance (Anderson et al., 2002; Singh, 2004). The two types of contours also differ in their effect upon attention, with attention tending to spread between inducing elements and completed regions only for the case of subjective contours, for which filling-in arises in the completed region (Davis \& Driver, 1997a; 1997b; but see Gold, Murray, Bennett, \& Sekuler, 2000 for different results). Furthermore, single cell recordings in primates (von der Heydt, Peterhans, \& Baumgartner, 1984), as well as neuroimaging data in human observers (Ffytche \& Zeki, 1996), suggest the involvement of low level visual processes common to both hemispheres, namely primary visual areas, particularly V2, in subjective contours. In contrast, the mechanism responsible for occlusion appears to involve higher level processes, lateralized to the right hemisphere (Corballis, Fendrich, Shapley, \& Gazzaniga 1999).

Another unsolved issue concerns the spatial limitations and scale dependency of both types of interpolation. Several studies have demonstrated a more salient perception of subjective contours when the configuration subtends a relatively small visual angle (e.g., Coren, 1972; Dumais \& Bradley, 1976; Gegenfurtner, Brown, \& Rieger, 1997; Kanizsa, 1976). Gerbino \& Kanizsa (1987) showed that elongation of a subjective blob depends on the retinal gap between endpoints.
Similar effects of retinal size were later observed for partially occluded shapes (Gerbino \& Fantoni, 2006).

There is, however, evidence suggesting a scale invariant perception of both subjective and occluded contours (Ringach \& Shapley, 1996; Shipley \& Kellman, 1992). For example, both the time needed for the production of a Kanizsa triangle (Kojo, Liinasuo, \& Rovamo, 1993) and the precision of the shape (Shipley \& Kellman, 1992) are largely unaffected by retinal size, but substantially enhanced by support ratio (i.e., the ratio of physically present contours length to interpolated contours length; see Figure 2B). However, these studies often used different shapes for subjective and occluded contours that differed in the amount of curvature (i.e., relatability), a factor that has been shown to critically affect interpolation strength (e.g., Shipley \& Kellman, 1992). Furthermore, the spatial arrangements of the visible edges varied across these different studies, and therefore the quantitative assessment of support ratio for subjective contours often differed from that for occluded contours (typically measured as proportion of occlusion). In some cases, such as circles occluded in a single region, support ratio complements the proportion of occlusion (i.e., a circle occluded in .25 of its contour has a support ratio of .75). In other cases, however, this comparison might not apply, as it is not always clear whether the "total edge length," as it is often referred to in the literature, includes all edges of the square or just those edges that are partially occluded (Guttman, Sekuler, \& Kellman 2003). It is therefore hard to draw conclusions about scale dependency of interpolation, and whether interpolation is limited equally by spatial factors in the case of subjective versus occluded contours. The first experiment examined these questions in adults.

A second purpose of the present study was to examine developmental trends in contour interpolation. This line of research provides contradictory findings regarding the age at which this perceptual skill of interpolation emerges. Measures of the age at which infants first interpolate partially occluded contours vary from 2 months (i.e., object unity; Johnson \& Aslin, 1995) to after 6 months of age (Otsuka, Kanazawa, \& Yamaguchi 2006). Measures of the age at which infants are first sensitive to subjective contours ranges from 3-4 months 
a
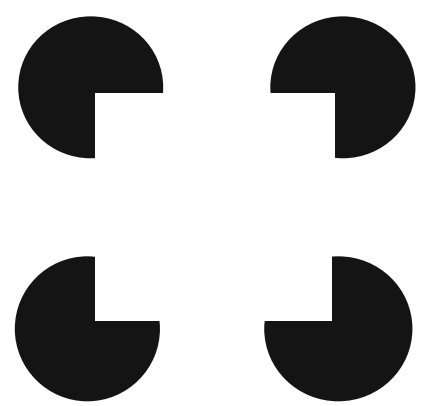

C

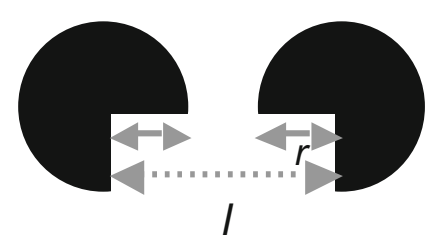

Fig. 2 a) Manipulation of support ratio and absolute size of the contour. b) Support ratio denotes the ratio of the two radii of the "pacmen" $(2 r)$ to the length of the side of the subjective square $(l)$ (adapted from Hadad,

(Ghim, 1990; Johnson \& Aslin, 1998; Kavsek, 2002; Otsuka et al., 2006; see also Sireteanu, 2000 for vernier offset lines) to after 5 months of age (e.g., Bertenthal, Campos, \& Haith, 1980; Condry, Smith, \& Spelke, 2001; Csibra, 2001; Treiber $\&$ Wilcox, 1980). Furthermore, studies testing the perceptual representation of a figure formed by subjective contours, specifically whether it has the properties of a real object acting as an occluding surface, provide evidence for this representation only at 8 months of age (Csibra, 2001). Consistent with this late onset of sensitivity to subjective contours, is the finding that only by 8 months of age do infants begin to show bindingrelated gamma oscillations similar to those of adults while perceiving a static Kanizsa square (Csibra, Davis, Spratling, $\&$ Johnson, 2000). Thus it appears that sensitivity to subjective contours develops later than sensitivity to occluded contours. Yet, in a direct comparison of the emergence of sensitivity to the two types of interpolation, Otsuka et al. (2006) showed the opposite pattern of results with sensitivity to subjective contours developing by 3-4 months and sensitivity to occluded contours developing much later - only after 6 months of age.

This literature also demonstrates substantial influence of spatial and temporal attributes on infants' response to interpolated contours. The perception of subjective contours during infancy depends on support ratio (Otsuka, Kanazawa, \& Yamaguchi, 2004), overall size (Johnson \& Aslin, 1995; 1996; Kavsek, 2002), and presence of motion cues (e.g., Curran, Braddick, Atkinson, Wattam-Bell, \& Andrew 1999; Jusczyk, Johnson, Spelke, \& Kennedy, 1999). Three- to 4month-olds respond to interpolated contours when the support $\mathrm{b}$

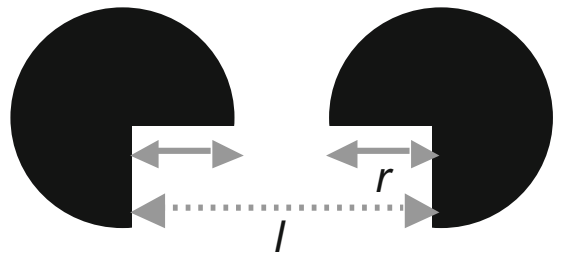

Maurer, \& Lewis, 2010). c) Overall smaller size of the display keeps the same support ratio $(2 r / l)$ but changes the absolute length of the interpolated contour $(l)$

ratio is high enough (Otsuka et al., 2004), or when motion information is available (Curran et al., 1999; Johnson \& Aslin, 1998). Furthermore, 4-month-olds respond to interpolated contours when the gap over which interpolation is required is relatively small but not when it is relatively large. This critical role of spatial separation between the inducers in infants' perception has been shown both for subjective (Kavsek, 2002) and occluded contours (e.g., Johnson \& Aslin, 1995,1996).

Although there is a large body of research on the onset of the ability to interpolate contours during the first year of life, there is little research on changes in this ability beyond infancy. To our knowledge, there are no studies on interpolation of occluded contours in children and only two studies on interpolation of subjective contours (Abravanel, 1982; Hadad, Maurer, \& Lewis, 2010a). Both studies found that interpolation continues to develop beyond infancy. In Abravanel's study, children's recognition accuracy of shapes formed by subjective contours improved from $60 \%$ to $100 \%$ between 3 and 5 years of age (Abravanel, 1982). Using an objective measurement, we showed that it is only by 12 years of age that children demonstrate adult-like interpolation of subjective contours (Hadad et al. 2010a). Interestingly, our data also revealed an increased effect of support ratio on interpolation with age. It is still unclear whether these developmental changes, found for subjective contours, reflect general immaturity in the interpolation mechanism underlying completion of any type of missing contours, or whether it is specific to subjective contours. Another unsettled issue concerns the way in which contour geometry may affect interpolation during early childhood. Our recent finding 
demonstrating lower sensitivity to support ratio at 6 years of age, as well as studies demonstrating the effect of the retinal size of the interpolated contours on interpolation during infancy, raise the possibility that the immature interpolation mechanism is more scale dependent. The second purpose of the present study was to trace the development of interpolation, and the way interpolation varies with contour geometry (i.e., support ratio and retinal size of the interpolated contours) in cases of both subjective and partially occluded contours.

In Experiment 1 we examined the effects of contour geometry on interpolation in adults. Specifically, a scale-dependent factor (i.e., retinal size) and a scale-independent factor (i.e., support ratio) were manipulated independently. In Experiment 2 we used a subset of contour retinal size and support ratio levels to compare interpolation of subjective and partially occluded contours in 6- and 9-year-olds to that in adults.

\section{Experiment 1: Contour interpolation in adults}

We examined adults' discrimination of shapes formed by interpolated contours as a function of the retinal size of the contour and the support ratio. To control for any differences between the two types of contours, we compared performance for subjective versus partially occluded contours when the interpolated shape, appearing at the front plane in the first case and at the back in the latter, was otherwise identical. Specifically, we ensured that interpolation occurred across the same four black inducers for both subjective and occluded stimuli.
A
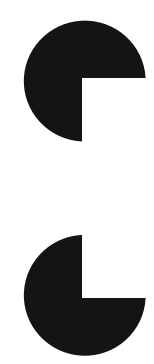
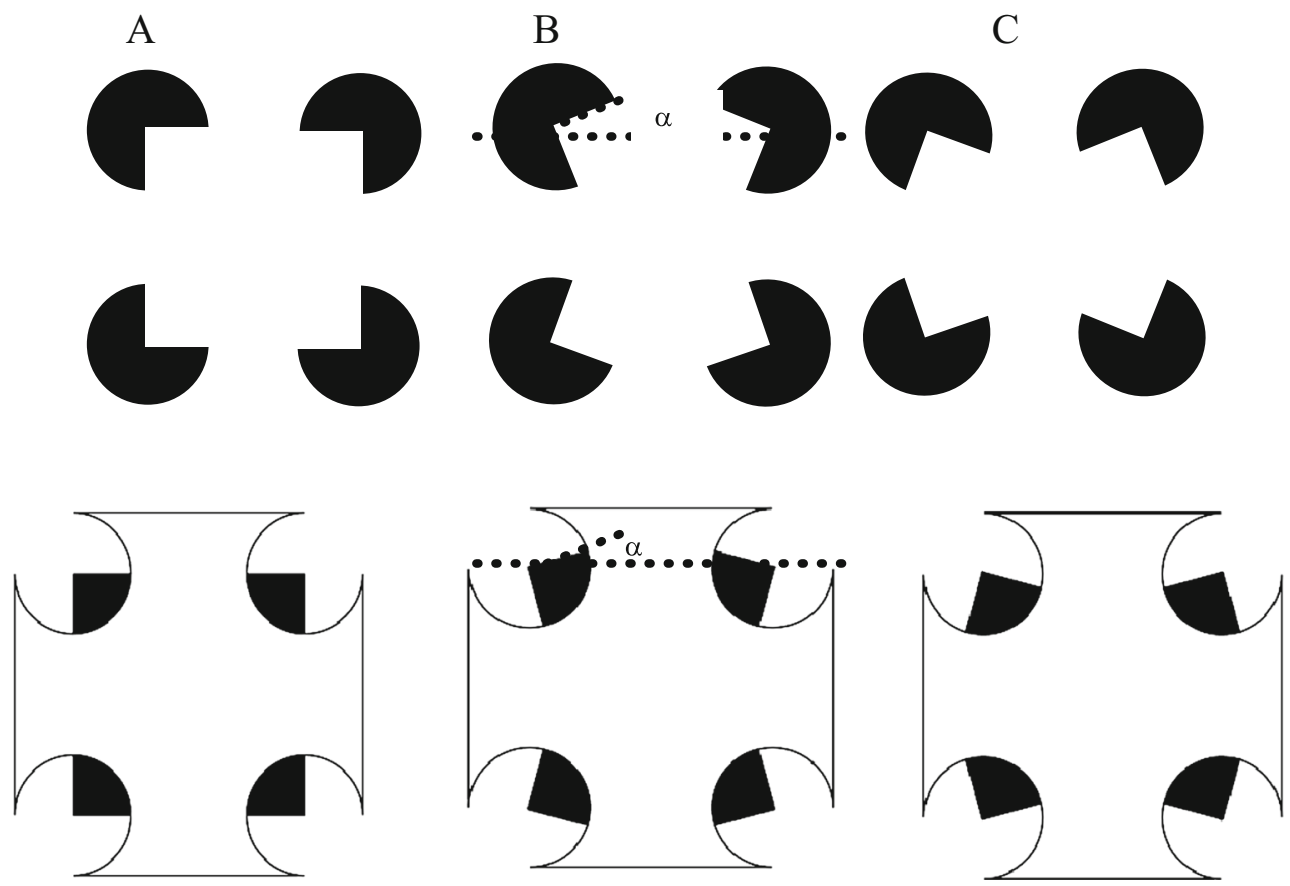

Fig. 3 Rotation of the inducers to produce fat or skinny shapes. Subjective contours are shown on the top and occluded contours on the bottom. $\alpha$ represents the rotation of the top-left inducer around the centre. procedure was used to vary $\alpha$ over trials
A shape discrimination task originally developed by Ringach \& Shapley (1996) was used. Observers discriminated between "fat" and "skinny" stimuli created by rotating the inducers (i.e., corners) of either a Kanizsa square or a partially occluded square (Fig. 3; the inducers in B and $\mathrm{C}$ are rotated to form a skinny subjective (top) and occluded (bottom) shape). Stimuli in the luminance-defined control conditions had parabolic contours or surfaces joining adjacent inducers to form the same shape produced by the subjective and occluded contours, respectively (Fig. 1).

We used a one-up, three-down staircase procedure (Levitt, 1971) to vary the angle of rotation of the inducing elements over trials. The amount of curvature (i.e., the "fatness" or "thinness") of the interpolated shape decreased as this angle of the inducing elements decreased over trials. Thresholds were defined as the minimum angle of rotation for which the observer could accurately discriminate the fat and the thin figures, and were thus taken to indicate precision of interpolation. Thresholds for the interpolated subjective and partially occluded contours were each compared to their luminance-defined counterparts to measure the strength of interpolation of contours into a shape.

\section{Method}

Participants The final sample consisted of 24 adults (mean age $=19.6$ years, range $=18-26$ years; 11 females) with normal or corrected-to-normal vision. Specifically, participants in the final sample had a linear letter acuity (Lighthouse Visual

Panel A shows the square with no rotation $(\alpha=0)$. In panel B, $\alpha>0$ produces skinny shapes. In panel $\mathrm{C}, \alpha<0$ produces fat shapes. A staircase 
Acuity Chart) of at least 20/20 in each eye with a maximum of -1 dioptre of optical correction (to rule out myopia greater than 1 dioptre, which would reduce vision at our testing distance of $100 \mathrm{~cm}$ ), worse acuity with a +3 dioptre add (to rule out hypermetropia greater than 3 dioptres), fusion at near on the Worth four dot test, and stereo acuity of at least 40 arcsec on the Titmus test. An additional four participants were excluded from the final sample for not passing visual screening.

Apparatus and stimuli Stimuli were generated on an Apple Macintosh Pro G5 computer using the MATLAB programming environment (version 7.4.0.287, MathWorks, Inc., Natick, MA, USA) and Psychophysics Toolbox (Brainard, 1997; Pelli, 1997). The stimuli were presented on a 21 -in color CRT monitor. Pixel resolution was $1600 \times 1200$, with one pixel corresponding to $0.015^{\circ}$ at the testing distance of 100 $\mathrm{cm}$, and the refresh rate was $85 \mathrm{~Hz}$. The mean luminance of the inducers (i.e., corners) was $4.55 \mathrm{~cd} / \mathrm{m}^{2}$, and the background was $90 \mathrm{~cd} / \mathrm{m}^{2}$. The contrast between the black elements and the white background was $90 \%$. Participants viewed the displays binocularly with their heads stabilized on a chin-rest.

Figure 1 shows examples of the stimuli. The partial occlusion stimuli had four visible black corners (Guttman \& Kellman, 2004). The subjective contour stimuli were composed of four black circles, each missing a $90^{\circ}$ notch, aligned to produce a subjective square, with the inducing edge of each precisely matched the length and location of the inducing edges in the corresponding partially occluded contours stimuli (see Table 1 for specific lengths for both types of stimuli as a function of support ratio and size). In the case of occluded contours, the stimuli contained a white cross-shaped inducer bounded by a 1.8 arcmin black contour $\left(20.4 \mathrm{~cd} / \mathrm{m}^{2}\right)$. The different sizes of the occluder in the different conditions are presented in Table 2. The segment removed from each corner had the shape of a half circle (diameters size are presented in Table 2), projected toward the associated corner with tangential extensions. The distance between two of the round notches at closest approach changed as a function of support ratio and size (see Table 2).

Displays in the luminance-defined condition (Fig. 1) precisely matched those of the subjective and partially occluded conditions, except that a luminance-defined contour, 6 pixels wide, was added in the former, and a luminance-defined surface in the latter. Specifically, an arc (in the case of the

Table 1 Length of inducing edge as a function of support ratio and size

\begin{tabular}{llll}
\hline & Large & Medium & Small \\
\hline High support (0.8) & $3.90 \mathrm{~cm}\left(2.23^{\circ}\right)$ & $2.39 \mathrm{~cm}\left(1.37^{\circ}\right)$ & $1.0 \mathrm{~cm}\left(0.57^{\circ}\right)$ \\
$\begin{array}{c}\text { Medium support } \\
(0.5)\end{array}$ & $2.40 \mathrm{~cm}\left(1.37^{\circ}\right)$ & $1.45 \mathrm{~cm}\left(0.83^{\circ}\right)$ & $0.60 \mathrm{~cm}\left(0.34^{\circ}\right)$ \\
Low support (0.2) & $1.40 \mathrm{~cm}\left(0.80^{\circ}\right)$ & $0.80 \mathrm{~cm}\left(0.46^{\circ}\right)$ & $0.30 \mathrm{~cm}\left(0.17^{\circ}\right)$
\end{tabular}


luminance-defined contours) or a surface (in the case of luminance-defined surface) with varying curvature, tangential to the black inducing edges at the points of tangent discontinuity, created the curved edges or surfaces of the fat and the skinny shapes.

The four inducers were rotated to produce fat and skinny shapes (Fig. 3B-C; Ringach \& Shapley, 1996). The skinny shape was formed by rotating the top-left and the lower-right inducers counterclockwise, and the lower-left and top-right inducers clockwise. Fat shapes were formed employing the opposite pattern of rotation.

The ratio between the two radii of the inducers $(2 r)$ and the length of the side of the square ( $l$; see Fig. $2 b$ ) defined the support ratio. This ratio was systematically varied by changing the size of inducing elements, while keeping the square size constant. Ratios of $80 \%, 50 \%$, and $20 \%$ were used. Each of these support ratios was presented in three different display sizes. Square size in the three conditions of support ratio subtended $1.26^{\circ}, 3.26^{\circ}$, or $5.37^{\circ}$ of visual angle for small, medium, and large displays, respectively. The diameter in the relatively high support ratio condition subtended $1.09^{\circ}, 2.55^{\circ}$, or $5.37^{\circ}$ of visual angle for small, medium, and large size, respectively, producing a support ratio of approximately $80 \%$.

The diameter in the medium support ratio condition subtended $0.66^{\circ}, 2.72^{\circ}$, or $1.64^{\circ}$ of visual angle for small, medium, and large size, respectively, producing a ratio of approximately $50 \%$, and the diameter in the low support ratio condition subtended $0.34^{\circ}, 0.63^{\circ}$, or $1.15^{\circ}$ of visual angle for small, medium, and large size, respectively, producing a ratio of $20 \%$. The luminance-defined contours were of medium support ratio and medium size.

Design The experiment employed an orthogonal combination of three factors, all manipulated within subject: contour type (subjective or partially occluded contour), support ratio (20\%, $50 \%$, and $80 \%$ ), and absolute size (small $\left(1.26^{\circ}\right)$, medium $\left(3.26^{\circ}\right)$, and large $\left(5.37^{\circ}\right)$ ).

Procedure The procedure was explained and informed consent was obtained from the participants. The experiment was conducted in accordance with the code of ethics of the World Medical Association (Declaration of Helsinki), and approval was given by the McMaster Research Ethics Board. Participants sat $100 \mathrm{~cm}$ from the monitor with their head stabilized in a chin-rest. Participants completed 20 tests: three support ratios $(20 \%, 50 \%$, and $80 \%$ ), and three sizes (small, medium, and large) for each of type of contour (subjective or partially occluded contour), as well as the corresponding luminance-defined conditions. Order of presentation for the type of contour (subjective or partially occluded contour) was counterbalanced across participants. Orders of support ratios were determined by a Latin Square. The order of testing for the three sizes was randomized within each support ratio.
Participants were instructed to fixate on a $1.15^{\circ}$ black circle in the centre of the screen at the beginning of each trial followed by a 250 -ms delay. The shape was then presented for 1000 ms. Participants judged whether the shape was fat or skinny, and the experimenter pressed the corresponding key. A one-up, three-down staircase procedure was used to vary the amount of curvature over trials, producing correct response rate equivalent to $79.4 \%$ accuracy (Levitt, 1971). A separate staircase was employed for each combination of support ratio and display size, with fat and skinny shapes appearing with equal probability and in random order in each. The first trial in each staircase contained a display of $10^{\circ}$ rotated inducers. After three consecutive correct responses, the angle of rotation was reduced by one octave to $5^{\circ}$. Following an error, the staircase reversed directions and a stimulus with a larger rotation angle was presented until three consecutive correct responses were made, after which the direction of the staircase reversed again to present successively smaller angles. The step size changed from one octave to half-octave intervals after two changes in the direction of the staircase ("reversals"). Testing ended after ten reversals, or after a maximum of 80 trials. For each observer, in each of the conditions, thresholds were calculated as the mean rotation angle of the final six reversals. Thresholds were defined as the minimum angle of rotation needed to accurately discriminate the interpolated shape as fat or skinny, and thus were taken to indicate interpolation precision.

Testing was preceded by demonstration trials, criterion trials, and a practice run with the same type of stimulus used in the test that was to follow. A full description of this procedure is presented in Hadad et al. (2010a, 2010b). Briefly, demonstration consisted of two trials, one with the fat and the other with the skinny shape, with $10^{\circ}$ rotations of the inducers employed in each. The skinny $10^{\circ}$ display was then repeated accompanied first by an example of positive feedback and then by an example of negative feedback. Subjects were then presented with criterion trials to verify they understood the task. These included a block of four trials with the same angle of rotation $\left(10^{\circ}\right)$ as the demonstration trials, with skinny and fat shapes presented in a random order. Participants had to judge correctly the shape on all four trials within a block, having three chances to meet this criterion in order to be included in the study. Observers then completed a practice run which was identical to the actual test. Data from the practice run was not included in the analyses. Participants completed the whole set of tests in one session that lasted approximately an hour, including breaks.

\section{Results}

Figure 4 shows performance on the shape discrimination task as a function of support ratio and retinal size, for each contour type. In a trimming procedure, each threshold was first 
a

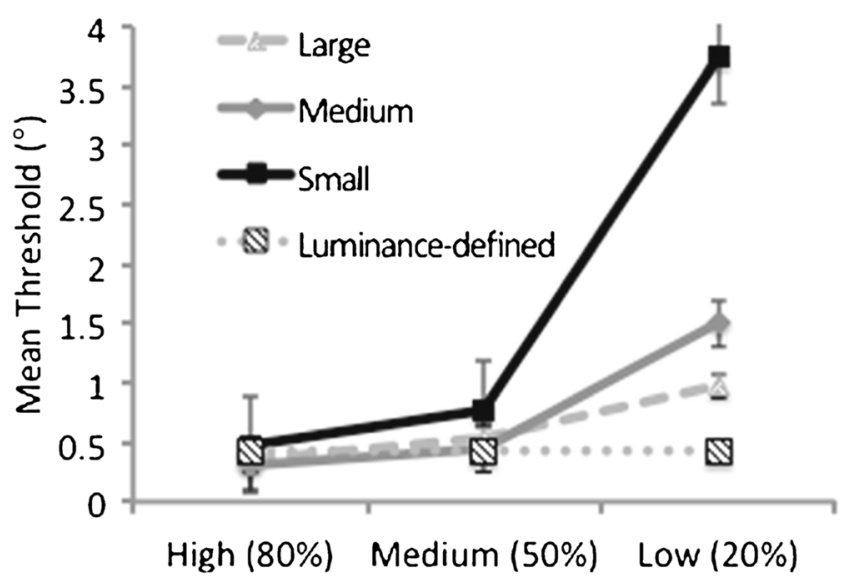

b

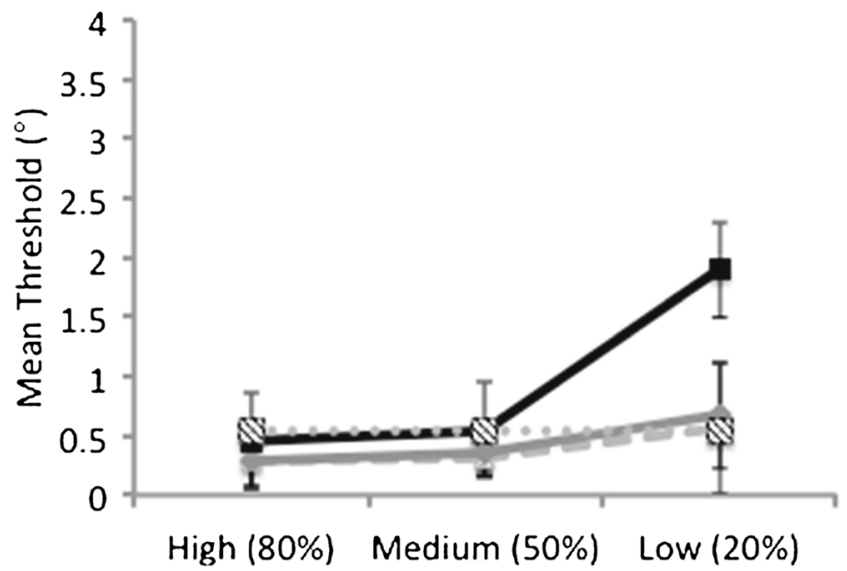

Fig. 4 Mean thresholds in degrees for (a) subjective and (b) partially occluded contours as a function of support ratio and retinal size. Bars indicate inferential confidence intervals, based on the method by Tryon and Lewis (2008)

converted to a $\mathrm{Z}$ score using the mean and standard deviation for a specific condition. $Z$ scores greater than +2.5 or less than -2.5 were replaced with the original group mean (see Kirk, 1990). Eleven data points were replaced, seven from subjective contours and four from occluded contours. Preliminary analyses revealed no significant effect of order of conditions, nor any interactions involving this factor, $F_{\mathbf{S}}<1$. The results were thus collapsed across this factor.

As expected, no significant difference was revealed between thresholds for the luminance-defined stimuli of the subjective and partially occluded shapes, $\mathrm{t}(23)=0.23, p>.82$, indicating that when no interpolation is required, shape discrimination is fairly similar across these two types of displays. This allowed us to attribute any differences observed in the interpolated conditions to the interpolation process itself, rather than to any general differences in the stimuli or in task difficulty.

A repeated measures ANOVA was carried out with support ratio $(80 \%, 50 \%$, and $20 \%$ ), retinal size (small, medium, and large), and contour type (subjective or partially occluded contour) as within-subject factors. The analyses revealed significant differences in thresholds obtained for subjective and occluded contours, $F(1,23)=25.60, p<0.001, \eta_{\mathrm{p}}^{2}=0.53$, indicating higher thresholds for subjective contours $(M=$ $1.01^{\circ}$ and $0.59^{\circ}$, for subjective and partially occluded contours, respectively). Interpolation of both types of contours varied with support ratio, $F(1,23)=35.51, p<0.001, \eta_{\mathrm{p}}^{2}=$ $0.61 ; F(1,23)=64.57, p<0.001, \eta_{\mathrm{p}}^{2}=0.74$, for subjective and occluded contours, respectively. However, a significant interaction between contour type and support ratio was also found, $\left.F(1,23)=17.63, p<0.001, \eta^{2}=0.43\right)$, suggesting a different pattern of the effect of support ratio on interpolation for the two types of contours. For subjective contours (see Fig. 4a), trend analysis revealed both linear and quadratic significant components, for all displays sizes (Large size: linear component: $F(1,22)=7.60, p<0.01, \eta_{\mathrm{p}}^{2}=0.25$; quadratic component: $F(1,22)=11.71, p<0.002, \eta_{\mathrm{p}}^{2}=0.34$; Medium size: linear component: $F(1,22)=9.39, p<0.006$, $\eta^{2}{ }_{\mathrm{p}}=0.30$; quadratic component: $F(1,22)=5.78, p<0.025$, $\eta_{\mathrm{p}}^{2}=0.20$; Small size: linear component: $F(1,22)=9.71, p<$ $0.005, \eta_{\mathrm{p}}^{2}=0.31$; quadratic component: $F(1,22)=23.03, p<$ $\left.0.0001, \eta_{\mathrm{p}}^{2}=0.51\right)$. This pattern reflects a substantial effect of support ratio throughout the different levels of this factor, with a more substantial effect, however, when support ratio decreased to $20 \%$. A significant interaction among contour type, support ratio, and retinal size, $F(1,23)=9.33, p<0.01, \eta_{\mathrm{p}}^{2}=$ 0.29 , further suggests this effect of support ratio increased for smaller display sizes. A different pattern was observed for partially occluded contours (see Fig. 4b). Trend analysis revealed that the function of the effect of support ratio in this case is better described by a quadratic trend, for all size conditions (significant quadratic components: Large size: $F(1$, $22)=9.67, p<0.005, \eta_{\mathrm{p}}^{2}=0.37$; Medium size: $F(1,22)=$ $51.39, p<0.0001, \eta_{\mathrm{p}}^{2}=0.70$; Small size: $F(1,22)=20.41, p$ $\left.<0.0001, \eta_{\mathrm{p}}^{2}=0.48\right)$. This indicates that for the case of partial occlusion the effect of support ratio originates mainly from the difference between medium and low support ratio. Altogether, these results suggest a more pronounced effect of support ratio for subjective contours, particularly, as can be seen in Fig. 4, for the smaller displays.

\section{Discussion}

The results from adults show that interpolation in both cases of subjective and partially occluded contours is tied more to support ratio than to retinal size. Over a rather large range of figure sizes, support ratio appears to be a reliable predictor of interpolation strength, with higher support ratios resulting in more precise contour representations. Although there appears to be an effect of retinal size on interpolation, it originates mainly from the smaller size of the displays (see Fig. 4 
and Shipley \& Kellman, 1992, for similar results). Furthermore, these effects of size, indicating better thresholds for large inducers, are in the opposite direction to previous effects reported in the literature (Dumais \& Bradley, 1976; Gerbino \& Fantoni, 2006). The extreme levels of support ratio and retinal size of displays (i.e., $20 \%$ support ratio and visual angle of $1.26^{\circ}$ ) resulted in extremely small inducers, possibly hindering interpolation and the curvature of the shape. This suggests that there might be a minimum amount of physically present contour required for proper interpolation to occur.

The finding of a stronger dependence of interpolation on support ratio than on retinal size for both types of contours is not surprising (Gillam, 1981; Shipley \& Kellman, 1992). This factor of support ratio is scale invariant, and contrary to the projected size of elements or the projected distance between elements, does not vary with the observer's distance from the object. Processes determining the unity of separated projected areas, such as interpolation of contours into bounded objects, are thus expected to depend more on support ratio, rather than on projected size. It should be noted, however, that larger absolute sizes than those used here could restrict interpolation even in adults, as evident in neurophysiologic studies in both animals (e.g., Kapadia, Ito, Gilbert, \& Westheimer, 1995; Stettler, Das, Bennett, \& Gilbert, 2002) and humans (e.g., Kapadia et al., 1995).

The present results also reveal systematic differences between interpolation of subjective and partially occluded contours into a shape. First, contour representations emerging from interpolation of subjective contours were less precise compared to those of occluded contours. Furthermore, there were differences in the spatial constraints of interpolation of subjective and partially occluded contours. For subjective contours, higher support ratios $(80 \%)$ were more precisely interpolated compared to medium support ratios (50\%), which were more precisely interpolated compared to the lower support ratios $(20 \%)$. However, for partially occluded contours, high and medium support ratios were interpolated with equal precision. It was only when support ratio was reduced to $20 \%$ that interpolation produced less precise representations. Combined, these data suggest that the effect of support ratio is more pronounced for subjective contours, and that the interpolation of the two types of the contours is not equally affected by the inducers' geometry.

Although these differences are largely inconsistent with the currently held version of the identity hypothesis, a possible interpretation of these differences might be that the two forms of completion do share a common mechanism, but this mechanism has a parameter that can take on different values and be reset, depending on the type of contours involved (Singh, 2004). In any case, these results indicate that interpolation in the mature visual system is mostly limited by scale independent factors (i.e., support ratio) and that these factors more substantially limit interpolation in the case of subjective contours. Experiment 2 tested spatial limitations of these two cases of interpolation in children.

\section{Experiment 2 - Development of contour interpolation}

Children aged 6 and 9 years and adults performed the same shape discrimination task as in Experiment 1, except that two levels of support ratio $(30 \%, 80 \%)$ and two levels of display sizes $\left(3.26^{\circ}, 5.37^{\circ}\right)$ were chosen from the nine conditions tested in Experiment 1. To maximize the effect of support ratio while collecting reliable data in our youngest age group, we increased the support ratio in the low support condition from $20 \%$ to $30 \%$. This experiment examined whether spatial constraints of interpolation change during childhood and determined the developmental stage at which these interpolation processes become adult-like.

\section{Method}

Participants Three age groups, 24 in each, participated in the experiment: 6 -year-olds (mean age $=5.48$ years, range $=$ 5.09-6.02 years, 11 female), 9-year-olds (mean age $=8.75$ years, range $=8.1-9.0$ years, 12 female), and adults (mean age $=19.20$ years, range $=18.01-22.04$ years, 11 female $)$.

All participants met our criteria on a visual screening examination. Visual screening was identical to that described in Experiment 1, except that 6-year-olds were tested using the Cambridge Crowding Cards. Six-year-olds had a linear letter acuity (Cambridge Crowding Cards) of at least 20/25 in each eye with a maximum of -1 dioptre of optical correction. Nine participants were excluded from the final sample: three 6year-olds (two of whom failed visual screening and one who did not complete both sessions of the experiment), one 9-yearold who failed visual screening, and five adults, all of whom failed visual screening. We obtained consent from the parents of the children and assent from the children themselves.

Apparatus and stimuli The apparatus and stimuli were identical to those used in Experiment 1 with the following exceptions. The diameter of the low support ratio condition was changed to $0.92^{\circ}$ and $1.6^{\circ}$ of visual angle, for medium and large sizes, respectively, giving rise to a ratio of $30 \%$.

Design The experiment employed orthogonal combinations of two factors, both manipulated within subject: contour type (subjective contours or partially occluded contours) and support ratio (30\% and $80 \%$ ). Absolute size was varied between subjects $\left(3.26^{\circ}\right.$ and $\left.5.37^{\circ}\right)$.

Procedure The procedure was identical to Experiment 1, with the following exceptions. Participants completed six tests on 
one display size $\left(3.26^{\circ}\right.$ or $\left.5.37^{\circ}\right)$ : two levels of support ratio (30\% and $80 \%$ ) for each contour type (subjective contour and occluded contour), as well as a corresponding luminancedefined condition. Six-year-olds participated in two 1-hour sessions within a 2-week period, whereas 9-year-olds and adults completed the procedure in a single 1-hour session.

\section{Results}

Mean raw thresholds for the shape discrimination task are presented on Tables 3 and 4, as a function of age and support ratio, for each type of contour and size. Twenty-eight data points were replaced by using the trimming procedure described in Experiment 1. Eight data points were replaced in adults and ten data points were replaced in 6- and 9-year-olds (five in each age group). Preliminary analyses revealed no significant effect of the order of the conditions, $F<1$, nor any interactions involving this factor. The results were thus collapsed across order.

We first examined age-related changes in the control conditions in which no interpolation was required, conducting a repeated measures ANOVA with sensitivity to luminancedefined contours (subjective control, occluded control) as a within-subject factor, and age as a between-subject factor. A significant effect of type of control condition, $F(1,142)=$ 22.86, $p<0.001, \eta_{\mathrm{p}}{ }^{2}=0.14$, indicated that thresholds for the luminance-defined contour (control for the subjective contours displays $)$ were higher $\left(\mathrm{M}=1.24^{\circ}\right)$ than thresholds for the luminance-defined surface $\left(\mathrm{M}=.95^{\circ}\right.$; control for the partially occluded displays). A significant main effect of age was found for luminance-defined conditions, $F(2,142)=119.62, p<$ $0.001, \eta_{\mathrm{p}}{ }^{2}=0.63$, as well as an interaction between the type of luminance-defined contour and age, $F(2,142)=18.19, p<$ $0.001, \eta_{\mathrm{p}}{ }^{2}=0.20$. Thresholds for both luminance-defined contours and luminance-defined surfaces improved with age, $F$ $(2,142)=99.58, p<0.001, \eta_{\mathrm{p}}{ }^{2}=0.58 ; F(2,142)=64.76, p<$ $0.001, \eta_{\mathrm{p}}{ }^{2}=0.47$, respectively. Inspection of Tables 3 and 4 suggests the interaction of age and contour type arose because

Table 3 Mean raw thresholds for the shape discrimination task for the large size displays, as a function of age and support ratio, for each type of contour

\begin{tabular}{llll}
\hline & 6 & 9 & Adults \\
\hline SC- High support & 2.618 & 0.694 & 0.357 \\
SC- Low support & 3.771 & 1.631 & 0.729 \\
PO- High support & 1.958 & 0.701 & 0.270 \\
PO- Low support & 3.051 & 1.047 & 0.464 \\
Luminance-defined contour & 2.561 & 0.783 & 0.491 \\
Luminance-defined surface & 1.921 & 0.702 & 0.575 \\
\hline
\end{tabular}

$S C$ subjective contour, $P O$ partially occluded contour
Table 4 Mean raw thresholds for the shape discrimination task for the medium size displays, as a function of age and support ratio, for each type of contour

\begin{tabular}{llll}
\hline & 6 & 9 & Adults \\
\hline SC- High support & 2.310 & 0.871 & 0.429 \\
SC- Low support & 3.641 & 1.552 & 0.988 \\
PO- High support & 2.021 & 0.475 & 0.372 \\
PO- Low support & 3.581 & 1.107 & 0.735 \\
Luminance-defined contour & 2.561 & 0.783 & 0.491 \\
Luminance-defined surface & 1.921 & 0.702 & 0.575 \\
\hline
\end{tabular}

$S C$ subjective contour, $P O$ partially occluded contour

the improvement after age 5 years was greater for luminancedefined contours than for luminance-defined surfaces. Posthoc comparisons showed that in both cases, performance reached adult-like levels at 9 years of age $(p s<.05)$.

Because of the differences observed between the control conditions of subjective and partially occluded contours and the differences documented across age, we calculated an index for interpolation cost for each condition. Specifically, this index was calculated by determining the difference between the interpolated threshold and the luminance-defined threshold divided by the luminance-defined threshold (see Eq. 1). The resulting index estimates the precision of the representation emerging from the interpolation, indicating the cost associated with interpolation when luminance-defined contours are not physically specified. Values statistically equal to zero indicate no cost for the interpolation of the contours into a shape. Higher values indicate a larger cost associated with interpolation.

Interpolation cost

$$
=\frac{\text { interpolation threshold-luminance defined threshold }}{\text { luminance-defined threshold }}
$$

Figure 5 shows means of interpolation cost as a function of age and support ratio, for each type of contour and size. A

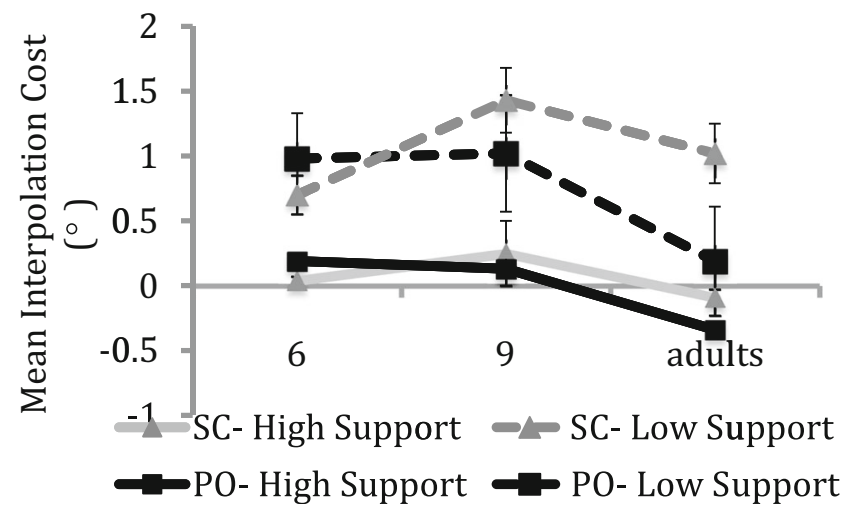

Fig. 5 Mean of interpolation cost (collapsed across display size) as a function of age and support ratio, for each type of contour 
repeated measures ANOVA was carried out with support ratio $(30 \%, 80 \%)$ and contour type (subjective contour, occluded contour) as within-subject factors, and age (6- and 9-year-olds and adults) and size $\left(3.26^{\circ}, 5.37^{\circ}\right)$ as between-subject factors. As expected, a significant effect of age was observed, $F(2$, 139) $=7.53, p<0.001, \eta_{\mathrm{p}}{ }^{2}=0.10$, as well as an effect of display size, $F(1,139)=7.08, p<0.001, \eta_{\mathrm{p}}{ }^{2}=0.09$, and of support ratio, $F(1,139)=168.71, p<0.0001, \eta_{\mathrm{p}}{ }^{2}=0.55$.

More importantly, the analysis revealed a significant twoway interaction between contour type (subjective, occluded contours) and age, $F(2,139)=4.29, p<0.002, \eta_{\mathrm{p}}{ }^{2}=0.06$, as well as a marginally significant interaction between contour type, age, and support ratio, $F(2,139)=2.86, p<0.06, \eta_{\mathrm{p}}{ }^{2}=$ 0.08 . For adults, interpolation cost varied between the two contour types, $F(1,47)=17.09, p<0.0001, \eta_{\mathrm{p}}{ }^{2}=0.27$, showing that while this cost was significantly different from zero in the case of subjective contours $(\mathrm{M}=.57 ; \mathrm{t}(48)=4.17, p<$ 0.001 ), there was no cost associated with interpolating occluded contours $(\mathrm{M}=-.07)$. In contrast, for 6- and 9-year-olds, there was cost associated with interpolating both subjective contours $(\mathrm{M}=0.39, \mathrm{M}=0.84$, respectively) and occluded contours $(\mathrm{M}=0.62, \mathrm{M}=0.63$, respectively). In fact, there were no significant differences in cost between the two cases of interpolation, for both the younger and the older groups of children, $F(1,46)=2.11, p>.15 ; F<1$, for 6- and 9-year-olds, respectively. Furthermore, for adults, the effect of support ratio interacted with contour type, $F(1,47)=$ 10.59, $p<0.02, \eta_{\mathrm{p}}{ }^{2}=0.18$, indicating again a more pronounced effect of support ratio for subjective contours compared to occluded contours (see Fig. 5). For children, in contrast, this effect of support ratio, $F(1$, $46)=50.20, p<0.0001, \eta_{\mathrm{p}}{ }^{2}=0.51 ; F(1,46)=52.68$, $p<0.0001, \eta_{\mathrm{p}}{ }^{2}=0.53$, for 6 - and 9-year-olds, respectively, did not vary between contour types, $F_{\mathrm{S}}<1$.

Interestingly, this three-way interaction between contour type, support ratio, and age group also suggests different developmental trajectories for the spatial constraints limiting interpolation in the two cases of contours (see Fig. 5). For the low support partially occluded displays, thresholds improved with age: specifically, cost decreased with age, $F(2,141)=$ $5.16, p<0.007, \eta_{\mathrm{p}}{ }^{2}=0.07$, with both 6 - and 9-year-old children showing higher cost than adults $(p \mathbf{s}<.05)$. For the low support subjective contours, on the other hand, age-related changes in thresholds displays show increments with age, $F$ $(2,141)=4.01, p<0.02, \eta_{\mathrm{p}}{ }^{2}=0.06$ (see Hadad et al., 2010a, for similar results), with post-hoc comparisons showing that interpolation cost at 9 years of age is higher than at 6 years $(p<$ .05 ), with an adult mean still slightly higher than at age 6 years. Altogether the results indicate different developmental trajectories for the interpolation of subjective and partially occluded contours as well as substantial changes in the spatial constraints of these two types of interpolation during childhood.

\section{Discussion}

Using a subset of levels of support ratio and absolute size of the contours, we replicated the pattern of results for adults obtained in Experiment 1, showing again that interpolation in adulthood is tied more to support ratio than to the absolute size of the interpolated contours. These spatial constraints were found for both types of contours. However, the results also indicate again that subjective contours are less precisely interpolated and interpolation in this case is more restricted by support ratio than in the case of partial occlusion. This pattern suggests that an identical initial interpolation mechanism might underlie the perception of subjective and occluded contours, but that other perceptual and cognitive processes must be involved, differently affecting the final representations of these two types of contours. We will go back to this point later in the General Discussion.

Experiment 2 further compared the interpolation of subjective and occluded contours by tracing their developmental courses, examining whether the spatial limitations of interpolation of these two types of contours differ at different points during development. The results demonstrated age-related changes in the spatial constraints on interpolation and different developmental trajectories for the two types of perceptual completion. Interpolation of both subjective and occluded contours was equally affected by spatial constraints during early childhood. However, while interpolation in the case of occlusion became more precise with age and less dependent on support ratio in adulthood, there was no consistent evidence for improvement with age in interpolating subjective contours (when computed in terms of interpolation cost) and their interpolation became even more tied to support ratio in adulthood. However, as this is the first direct comparison of interpolation of the two types of contours across age, these conclusions about the differential developmental trends for subjective and occluded contours must await further investigation.

The effect shown here of contour geometry on interpolation in young childhood is in agreement with the effect of spatial distances shown in contour integration studies (Kovacs et al., 1999; Hadad et al. 2010a, 2010b). Both interpolation in the cases of subjective and occluded contours and integration of fragmented elements into a contour is critically restricted during childhood by the spatial distance over which this perceptual process is employed, with both cases of contour perception extending over nearly the same spatial distances. It has been shown that long-range horizontal interactions in V1 mediating spatial integration can be augmented over a number of nodes in a network of connections, as long as the spacing between elements is kept within a critical distance even in adults (e.g., Stettler, Das, Bennett, \& Gilbert, 2002). These long-range facilitatory interactions between orientation-tuned spatial channels that seem to span shorter 
distances in children than in adults (e.g., Kovacs, Kozma, Feher, \& Benedek, 1999) may underlie children's performance both in the case of contour interpolation and in the contour integration task.

Visual perception of objects depends on interpolation and grouping processes that often act on fragmentary and missing input. The rather protracted development that we found here for this basic ability of contour interpolation may underlie a group of visual functions with protracted developmental sequences. Each of these visual functions involves interpolation and integration among elements into a global visual pattern. These include the ability to group fragmented contours into a coherent shape (Hadad \& Kimchi, 2006), the detection of a global form in Glass patterns (Lewis et al., 2002), configural face processing (Mondloch, Le Grand, \& Maurer, 2002; but see Crookes \& McKone, 2009), and configural processing of hierarchical patterns (Burack, Iarocci, \& Randolph, 2000; Kimchi et al., 2005; Mondloch, Geldart, Maurer, \& de Schonen, 2003). This delayed maturation of perceptual processes underlying object perception may be explained, at least in part, by immature contour interpolation processes which connect visible contours across gaps to produce units that correspond more accurately to the actual objects in a scene.

\section{General discussion}

A large body of empirical evidence as well as logical arguments have been accumulated to support the notion of a common underlying interpolation mechanism which produces precise and, according to this notion, identical representations of contours in both subjective and occluded interpolation cases (Kellman, Temesvary, Palmer, \& Shipley, 2000; Kellman et al., 1998). The identity hypothesis suggests a local-based computational process by which interpolation processes operate (for more details, see Kellman, 2003; Kellman, Guttman, \& Wickens, 2001; Kellman \& Shipley, 1991). Specifically, tangent discontinuity, places at intersections or junctions in the stimuli where contours have no unique orientation, have been defined as points at which computations subserving interpolation begin. It has been proposed that cells sensitive to the contours that end in their receptive fields in the visual cortex (often called "end-stopped" cells) underlie the detection of these junctions (e.g., Heitger, Rosenthaler, von der Heydt, Peterhans, \& Kübler 1992). This local mechanism is strongly governed by contour relatability, such that contours that are smooth and monotonic, and at their endpoints match the slopes of real contours, are more readily interpolated (Kellman \& Shipley, 1991). Given this critical role of relatability, some tolerance around collinearity early in life, with gradual decrease in this tolerance as perceptual experience accrues (Hadad et al., 2010b), might underlie the agerelated changes in interpretation observed here.
Several neural models of contour interpolation have been proposed (e.g., Grossberg \& Mingolla, 1985; Heitger, von der Heydt, Peterhans, Rosenthaler, \& Kübler 1998; reviewed in Kellman, 2003). It has been suggested that physically present edges on either side of the interpolated gap trigger nonlinear grouping operators mediated by bipole cells (Heitger et al., 1998). Computations subserving interpolation may also be mediated by an interactive network of orientation-signaling units generating facilitative horizontal interactions triggered by successive activation of adjacent edge sensitive neurons (Field, Hayes, \& Hess, 1993).

Although studied separately, the processes of contour integration and interpolation of fragmented contours are both likely to be mediated by these possible neural mechanisms. Consistent with this suggestion, the two different literatures demonstrate similar restrictions of contour geometry and scale-dependence of these perceptual mechanisms. In both cases, for example, it has been shown that the mature mechanism in adults is tied more to scale-invariant factors (e.g., Shipley \& Kellman, 1992; Sigman, Cecchi, Gilbert, \& Magnasco, 2001) and the developing mechanism spans shorter spatial distances compared to those in adults (e.g., Kovács, 2000; Hadad, Maurer, \& Lewis, 2010b). These psychophysical findings have been steadily gaining neurophysiological support. Responses of V1 neurons are modulated by the joint presence of contour elements within the receptive field and its surround (e.g., Kapadia, Ito, Gilbert, \& Westheimer, 1995). Interactions between these neurons depends on the precise geometrical arrangement of segments and through these interactions neural responses become selective for combinations of stimulus characteristics that can extend far from the receptive field core (Sigman et al., 2001). The nature of these interactions is determined by contour geometry and visual characteristics that have been shown to affect both contour interpolation and integration of segments into a contour, such as the spatial arrangement and the orientation of the segments within the visual scene. For these different perceptual phenomena, processing depends on a precise geometrical arrangement of the segments in a manner corresponding to the specificity of linkage of cortical columns by long-range horizontal connections. It is possible then that the perceptual organization of segments into completed coherent contours in the different cases of fragmented, occluded, and subjective contours share common neuronal mechanism involving horizontal intrinsic connections in V1 (e.g., Spillmann \& Werner, 1996). The final percept and the different phenomenology arising from these different cases could result from possible reentrant feedback projections diverging to cells at lower levels which respond selectively to specific properties of the visual input. These preliminary speculations, however, are beyond the scope of the current work, and thus must await further investigation.

These neural mechanisms take time to develop. Functioning of the "end-stopped" cells in visual cortex 
involved in junction detection has been shown to undergo substantial development (e.g., Pack, Livingstone, Duffy, \& Born, 2003). Long-range facilitatory integrations between orientationtuned spatial channels become adult-like only towards the end of childhood (Kovács, 1996, 2000). Cortical connectivity in Layers 2 and 3 of V1 seems to be immature even at 5 years of age (Burkhalter, Bernardo, \& Charles, 1993), and the development of feedback connections between V1 and V2 also seems delayed (Burkhalter, 1993). These later developing connections have been proposed as part of the physiological mechanism enabling the improvement with age in interpolating and integrating fragmented input (Kovács, 1996, 2000).

However, there is more than one process at work in perceptual completion. In addition to the relatively local interpolation process based on contour relatability, there are other, more global processes involved. Contrary to the large body of evidence for local mechanism of interpolation demonstrating, for example, that interpolation works equally well when the outcomes are familiar or unfamiliar, symmetric or asymmetric (e.g., Kellman, 2003), global cues have often been shown to be important. Evidence from priming studies, for example, has demonstrated both local and global effects in interpolation (Sekuler, Palmer, \& Flynn, 1994; van Lier, van der Helm, \& Leeuwenberg, 1995). However, the role of these global cues seems different in the two cases of interpolated contours: only in an occluded display are observers aware that part of an object is hidden from view. The two types of completion also differ in basic aspects of scene representation, such as their depth relations with adjacent surfaces. Cognitive processes may thus play a more substantial role in the case of partial occlusion. Consistent with this hypothesis is the work showing that occluded contours can be completed even in a patient with visual agnosia, who is impaired in both binding of contours to shapes and in the computation of figure-ground relations in overlapping shapes (Giersch, Humphreys, Boucart, \& Kovács, 2000). This implies that although computations of contours between collinear segments are based on long-range connections within early visual areas (e.g., Gilbert \& Wiesel, 1989), they are likely to be supported by higher-level processes (Lesher, 1995). Age-related changes in these cognitive processes and their possible role in perceptual completion may explain the present results: partially occluded and subjective contours seem similar both in the precision of the interpolated contours (thresholds) and in the spatial constraints on their interpolation during early childhood. With age, cognitive processes might come into play in a more substantial way for partial occlusion leading to greater precision of the interpolated representations and a reduced influence of geometrical factors such as support ratio.

Different processes seem to underlie the age-related changes in the interpolation of subjective contours than those responsible for the changes for partial occlusion. At all ages, sensitivity to subjective contours was better with higher support ratio than with lower support ratio. However, the effect of support ratio increased with age (see Hadad et al., 2010a, for similar results). These results suggest that, during middle childhood, the interpolation of subjective contours becomes tied to the support ratio, so that contours that are more likely to reflect real objects' contours (i.e., highly supported contours) are more readily interpolated (Hadad et al., 2010a). Contrary to the visual angle formed by the absolute length of the interpolated contour that varies with distance ( $l$ in Fig. 2b), this ratio of the physically specified contours to the total physically specified contours $(2 r / l$ in Fig. 2b) is invariant across viewing distances. In other words, strength of interpolation of a single object with a given support ratio remains constant across different distances, allowing a single object to be perceived as a bounded entity regardless of its distance from the observer. Our results suggest that this scale-invariant mechanism, allowing perceptual constancy and unified perception of objects, evolves gradually during development. Developmental studies on size constancy in children are consistent with this interpretation showing, for example, that 5- and 6-year-olds underestimate the size of a distant object, and it is only at 9 years of age that children exhibit size constancy for distant objects (Granrud \& Schmechel, 2006). The increased sensitivity of the visual system with age to scale-invariant factors, such as support ratio, might be a result of statistical learning of natural contours accumulating over the years. The visual system may increasingly use this information on natural contours in perceiving the unity of objects, designating highly supported contours as better candidates for composing a single bounded object than contours with lower support ratios.

The differential age-related changes in the two types of visual completion might also be attributed to the difference in the rate of occurrence of these two perceptual events in real-world settings. Given that the environmental conditions that give rise to camouflage are much more restricted than those that give rise to partial occlusion (e.g., Anderson et al., 2002), it is reasonable to expect that the visual system has developed first a mechanism to transcend input fragmentation arising from partial occlusion, urging perceptual completion abilities to attain the ultimate level of functioning in a relatively little time.

\section{References}

Abravanel, E. (1982). Perceiving subjective contours during early childhood. Journal of Experimental Child Psychology, 33(2), 280-287.

Anderson, B. L., Singh, M., \& Fleming, R. W. (2002). The interpolation of object and surface structure. Cognitive Psychology, 44(2), 148-190.

Bertenthal, B. I., Campos, J. J., \& Haith, M. M. (1980). Development of visual organization: The perception of subjective contours. Child Development, 51, 1072-1080.

Brainard, D. H. (1997). The psychophysics toolbox. Spatial Vision, 10, 433-436. 
Burack, J. A., Enns, J. T., Iarocci, G., \& Randolph, B. (2000). Age differences in visual search for compound patterns: long-versus shortrange grouping. Developmental Psychology, 36(6), 731.

Burkhalter, A. (1993). Development of forward and feedback connections between areas V1 and V2 of human visual cortex. Cerebral Cortex, 3(5), 476-487.

Burkhalter, A., Bernardo, K. L., \& Charles, V. (1993). Development of local circuits in human visual cortex. The Journal of Neuroscience, 13(5), 1916-1931.

Condry, K. F., Smith, W. C., \& Spelke, E. S. (2001). Development of perceptual organization. Emerging Cognitive Abilities in Early Infancy, 1, 1-28.

Corballis, P. M., Fendrich, R., Shapley, R. M., \& Gazzaniga, M. S. (1999). Illusory contour perception and amodal boundary completion: evidence of a dissociation following callosotomy. Journal of Cognitive Neuroscience, 11(4), 459-466.

Coren, S. (1972). Subjective contours and apparent depth. Psychological Review, 79(4), 359

Crookes, K., \& McKone, E. (2009). Early maturity of face recognition: No childhood development of holistic processing, novel face encoding, or face-space. Cognition, 111(2), 219-247.

Csibra, G. (2001). Illusory contour figures are perceived as occluding surfaces by 8-nonth-old infants. Developmental Science, 4(4), F7F11.

Csibra, G., Davis, G., Spratling, M. W., \& Johnson, M. H. (2000). Gamma oscillations and object processing in the infant brain. Science, 290(5496), 1582-1585.

Curran, W., Braddick, O. J., Atkinson, J., Wattam-Bell, J., \& Andrew, R. (1999). Development of illusory-contour perception in infants. Perception-London, 28, 527-538.

Davis, G., \& Driver, J. (1997a). Spreading of visual attention to modally versus amodally completed regions. Psychological Science, 8(4), 275-281.

Davis, G., \& Driver, J. (1997b). A functional role for illusory colour spreading in the control of focused visual attention. PerceptionLondon, 26, 1397-1412.

Davis, G., \& Driver, J. (1998). Kanizsa subjective figures can act as occluding surfaces at parallel stages of visual search. Journal of Experimental Psychology. Human Perception and Performance, 24(1), 169

Dumais, S. T., \& Bradley, D. R. (1976). The effects of illumination level and retinal size on the apparent strength of subjective contours. Perception \& Psychophysics, 19(4), 339-345.

ffytche, D. H., \& Zeki, S. (1996). Brain activity related to the perception of illusory contours. NeuroImage, 3, 104-108.

Field, D. J., Hayes, A., \& Hess, R. F. (1993). Contour integration by the human visual system: Evidence for a local "association field". Vision Research, 33(2), 173-193.

Gegenfurtner, K. R., Brown, J. E., \& Rieger, J. (1997). Interpolation processes in the perception of real and illusory contours. Perception-London, 26, 1445-1458.

Gerbino, W., \& Fantoni, C. (2006). Visual interpolation is not scale invariant. Vision Research, 46(19), 3142-3159.

Gerbino, W., \& Kanizsa, G. (1987). Can we see constructs? In The perception of illusory contours (pp. 246-252). New York: Springer.

Ghim, H. R. (1990). Evidence for perceptual organization in infants: Perception of subjective contours by young infants. Infant Behavior and Development, 13(2), 221-248.

Giersch, A., Humphreys, G. W., Boucart, M., \& Kovács, I. (2000). The computation of occluded contours in visual agnosia: Evidence for early computation prior to shape binding and figure-ground coding. Cognitive Neuropsychology, 17(8), 731-759.

Gilbert, C. D., \& Wiesel, T. N. (1989). Columnar specificity of intrinsic horizontal and corticocortical connections in cat visual cortex. The Journal of Neuroscience, 9(7), 2432-2442.
Gillam, B. (1981). Separation relative to length determines the organization of two lines into a unit. Journal of Experimental Psychology. Human Perception and Performance, 7(4), 884.

Gold, J. M., Murray, R. F., Bennett, P. J., \& Sekuler, A. B. (2000). Deriving behavioural receptive fields for visually completed contours. Current Biology, 10(11), 663-666.

Granrud, C. E., \& Schmechel, T. T. (2006). Development of size constancy in children: a test of the proximal mode sensitivity hypothesis. Perception \& Psychophysics, 68(8), 1372-1381.

Grossberg, S., \& Mingolla, E. (1985). Neural dynamics of form perception: boundary completion, illusory figures, and neon color spreading. Psychological Review, 92(2), 173-211.

Guttman, S. E., \& Kellman, P. J. (2004). Contour interpolation revealed by a dot localization paradigm. Vision Research, 44(15), 1799 1815.

Guttman, S. E., Sekuler, A. B., \& Kellman, P. J. (2003). Temporal variations in visual completion: A reflection of spatial limits? Journal of Experimental Psychology. Human Perception and Performance, 29(6), 1211-1227.

Hadad, B. S., \& Kimchi, R. (2006). Developmental trends in utilizing perceptual closure for grouping of shape: Effects of spatial proximity and collinearity. Perception \& Psychophysics, 68(8), 1264-1273.

Hadad, B. S., Maurer, D., \& Lewis, T. L. (2010a). The development of contour interpolation: Evidence from subjective contours. Journal of Experimental Child Psychology, 106(2), 163-176.

Hadad, B. S., Maurer, D., \& Lewis, T. L. (2010b). The effects of spatial proximity and collinearity on contour integration in adults and children. Vision Research, 50(8), 772-778.

He, Z. J., \& Nakayama, K. (1992). Surfaces versus features in visual search. Nature, 359(6392), 231-233.

Heitger, F., Rosenthaler, L., von der Heydt, R., Peterhans, E., \& Kübler, O. (1992). Simulation of neural contour mechanisms: from simple to end-stopped cells. Vision Research, 32(5), 963-981.

Heitger, F., von der Heydt, R., Peterhans, E., Rosenthaler, L., \& Kübler, O. (1998). Simulation of neural contour mechanisms: representing anomalous contours. Image and Vision Computing, 16(6), 407-421.

Johnson, S. P., \& Aslin, R. N. (1995). Perception of object unity in 2month-old infants. Developmental Psychology, 31(5), 739.

Johnson, S. P., \& Aslin, R. N. (1996). Perception of object unity in young infants: The roles of motion, depth, and orientation. Cognitive Development, 11(2), 161-180.

Johnson, S. P., \& Aslin, R. N. (1998). Young infants' perception of illusory contours in dynamic displays. Perception-London, 27, 341354.

Jusczyk, P. W., Johnson, S. P., Spelke, E. S., \& Kennedy, L. J. (1999). Synchronous change and perception of object unity: Evidence from adults and infants. Cognition, 71(3), 257-288.

Kanizsa, G. (1976). Subjective contours. Scientific American, 234(4), 48 52.

Kapadia, M. K., Ito, M., Gilbert, C. D., \& Westheimer, G. (1995). Improvement in visual sensitivity by changes in local context: parallel studies in human observers and in V1 of alert monkeys. Neuron, 15(4), 843-856.

Kavsek, M. J. (2002). The perception of static subjective contours in infancy. Child Development, 73, 331-344.

Kellman, P. J. (2003). Interpolation processes in the visual perception of objects. Neural Networks, 16(5), 915-923.

Kellman, P. J., Guttman, S. E., \& Wickens, T. D. (2001). Geometric and neural models of object perception. Advances in Psychology, 130, 183-245.

Kellman, P. J., \& Shipley, T. F. (1991). A theory of visual interpolation in object perception. Cognitive Psychology, 23(2), 141-221.

Kellman, P. J., Temesvary, A., Palmer, E. M., \& Shipley, T. F. (2000). Separating local and global processes in object perception: Evidence from an edge localization paradigm. Investigative Ophthalmology \& Visual Science, 41(4), S741-S741. 
Kellman, P. J., Yin, C., \& Shipley, T. F. (1998). A common mechanism for illusory and occluded object completion. Journal of Experimental Psychology. Human Perception and Performance, 24(3), 859-869.

Kimchi, R., Hadad, B., Behrmann, M., \& Palmer, S. E. (2005). Microgenesis and ontogenesis of perceptual organization evidence from global and local processing of hierarchical patterns. Psychological Science, 16(4), 282-290.

Kirk, R. E. (1990). Statistics: an Introduction. Texas: Holt, Rinehart, and Winston. Inc.

Kojo, I., Liinasuo, M., \& Rovamo, J. (1993). Spatial and temporal properties of illusory figures. Vision Research, 33(7), 897-901.

Kovács, I. (1996). Gestalten of today: early processing of visual contours and surfaces. Behavioural Brain Research, 82(1), 1-11.

Kovács, I. (2000). Human development of perceptual organization. Vision Research, 40(10), 1301-1310.

Kovacs, I., Kozma, P., Feher, A., \& Benedek, G. (1999). Late maturation of visual spatial integration in humans. Proceedings of the National Academy of Sciences, 96(21), 12204-12209.

Lesher, G. W. (1995). Illusory contours: Toward a neurally based perceptual theory. Psychonomic Bulletin \& Review, 2(3), 279-321.

Levitt, H. (1971). Transformed up-down methods in psychoacoustics. The Journal of the Acoustical Society of America, 49(2B), 467-477.

Lewis, T. L., Ellemberg, D., Maurer, D., Wilkinson, F., Wilson, H. R., Dirks, M., \& Brent, H. P. (2002). Sensitivity to global form in glass patterns after early visual deprivation in humans. Vision Research, 42(8), 939-948.

Michotte, A., Thines, G., \& Crabbe, G. (1991/1964). Amodal completion of perceptual structures. In G. Thines, A. Costall, \& G.Butterworth (Eds.), Michotte's experiment al phenomenology of perception (pp. 140-167). Hillsdale, NJ: Erlbaum. [Original work published in 1964].

Mondloch, C. J., Geldart, S., Maurer, D., \& de Schonen, S. (2003). Developmental changes in the processing of hierarchical shapes continue into adolescence. Journal of Experimental Child Psychology, 84(1), 20-40.

Mondloch, C. J., Le Grand, R., \& Maurer, D. (2002). Configural face processing develops more slowly than featural face processing. Perception-London, 31(5), 553-566.

Murray, M. M., Foxe, D. M., Javitt, D. C., \& Foxe, J. J. (2004). Setting boundaries: brain dynamics of modal and amodal illusory shape completion in humans. The Journal of Neuroscience, 24(31), 6898-6903.

Nakayama, K., \& Shimojo, S. (1992). Experiencing and perceiving visual surfaces. Science, 257(5075), 1357-1363.

Otsuka, Y., Kanazawa, S., \& Yamaguchi, M. K. (2004). The effect of support ratio on infants' perception of illusory contours. Perception-London, 33(7), 807-816.
Otsuka, Y., Kanazawa, S., \& Yamaguchi, M. K. (2006). Perceptual transparency in 3- to 4-month-old infants. Perception, 35(12), 16251636.

Pack, C. C., Livingstone, M. S., Duffy, K. R., \& Born, R. T. (2003). Endstopping and the aperture problem: two-dimensional motion signals in macaque V1. Neuron, 39(4), 671-680.

Pelli, D. G. (1997). The VideoToolbox software for visual psychophysics: Transforming numbers into movies. Spatial Vision, 10(4), 437-442.

Rensink, R. A., \& Enns, J. T. (1998). Early completion of occluded objects. Vision Research, 38(15), 2489-2505.

Ringach, D. L., \& Shapley, R. (1996). Spatial and temporal properties of illusory contours and amodal boundary completion. Vision Research, 36(19), 3037-3050.

Sekuler, A. B., Palmer, S. E., \& Flynn, C. (1994). Local and global processes in visual completion. Psychological Science, 5(5), 260 267.

Shipley, T. F., \& Kellman, P. J. (1992). Strength of visual interpolation depends on the ratio of physically specified to total edge length. Perception \& Psychophysics, 52(1), 97-106.

Sigman, M., Cecchi, G. A., Gilbert, C. D., \& Magnasco, M. O. (2001). On a common circle: natural scenes and Gestalt rules. Proceedings of the National Academy of Sciences, 98(4), 1935-1940.

Singh, M. (2004). Modal and amodal completion generate different shapes. Psychological Science, 15(7), 454-459.

Sireteanu, R. (2000). Texture segmentation, "pop-out”, and feature binding in infants and children. In C. Rovee-Collier, L. Lipsitt, \& H. Hayne (Eds.), Progress in infancy research (Vol. 1, pp. 183-250). Mahwah, NJ: Lawrence Erlbaum.

Spillmann, L., \& Werner, J. S. (1996). Long-range interactions in visual perception. Trends in Neurosciences, 19(10), 428-434.

Stettler, D. D., Das, A., Bennett, J., \& Gilbert, C. D. (2002). Lateral connectivity and contextual interactions in macaque primary visual cortex. Neuron, 36(4), 739-750.

Treiber, F., \& Wilcox, S. (1980). Perception of a" subjective" contour by infants. Child Development, 51, 915-917.

Tryon, W. W., \& Lewis, C. (2008). An inferential confidence interval method of establishing statistical equivalence that corrects Tryon's (2001) reduction factor. Psychological Methods, 13(3), 272.

van Lier, R. J., van der Helm, P. A., \& Leeuwenberg, E. L. J. (1995). Competing global and local completions in visual occlusion. Journal of Experimental Psychology. Human Perception and Performance, 21(3), 571-583.

von der Heydt, R., Peterhans, E., \& Baumgartner, G. (1984). Illusory contours and cortical neuron responses. Science, 224(4654), 12601262.

Yin, C., Kellman, P. J., \& Shipley, T. F. (1997). Surface completion complements boundary interpolation in the visual integration of partly occluded objects. Perception-London, 26, 1459-1479. 